\title{
La Grande Galerie du Château de Berlin LE LIEU, SON AMÉNAGEMENT ET SON EMPLOI AU COURS DE TROIS SIÈCLES
}

\author{
Guido Hinterkeuser
}

L'idée de la Grande Galerie ${ }^{1}$ (Ill. 1) du château de Berlin a pris forme à partir de 1708 , dans les dernières années de régence du premier roi de Prusse ${ }^{2}$ Frédéric ${ }^{\text {er }}$. La construction put commencer après que la décision eut été prise en 1706-1707 de doubler la surface du château baroque vers l'ouest et de flanquer la cour extérieure d'ailes comportant un rez-dechaussée, deux étages et un attique. Succédant à Andreas Schlüter, responsable de la construction du château jusque-là, l'architecte suédois Johann Friedrich Eosander prit en charge la responsabilité de la conception, de l'édification et de l'aménagement des nouveaux corps de bâtiment ${ }^{3}$. C'est donc à Eosander que l'on doit l'idée de cette galerie orientée vers le Lustgarten (jardin d'agrément), et directement alignée sur l'enfilade des salles d'apparat tout juste achevées par Schlüter (Ill. 2).

1. Le nom de große Gallerie n'apparaît que rarement dans les documents (voir par exemple dans le document cité dans la note 33). La Grande Galerie est plus connue sous les noms de Bildergalerie, de Gemäldegalerie ou encore de Cobelingalerie, qui du reste, comme il sera montré dans la suite, n'ont eu cours qu'à des périodes précises. C'est pourquoi nous nous en tiendrons pour la suite au terme de Grande Galerie.

2. Klünner, Peschken, 1982 : 485-488; Wiesinger, $1992:$ 186-191; Geyer, 1992, vol. texte : 16-18; Peschken, Wiesinger, 2001, vol. texte : 195-199, 331-340 et vol. illustrations : ill. $\mathrm{n}^{\mathrm{os}} 329-349$.

3. Geyer, 1992, vol. texte : 8-15; Holland, $2002:$ 215-239. 


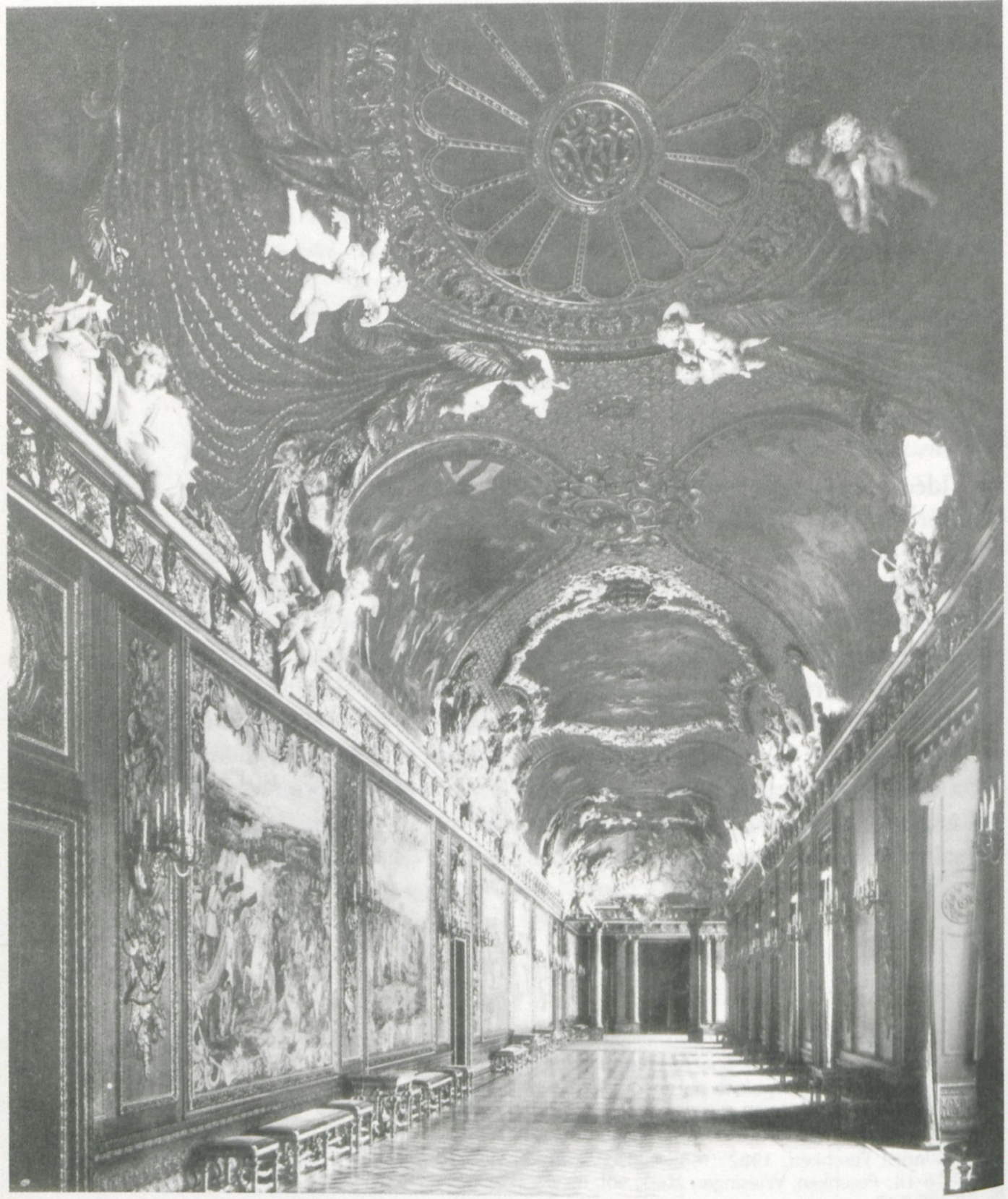

III. 1. Château de Berlin, Grande galerie (alors Galerie des Gobelins), vue vers I'ouest, 1916. 


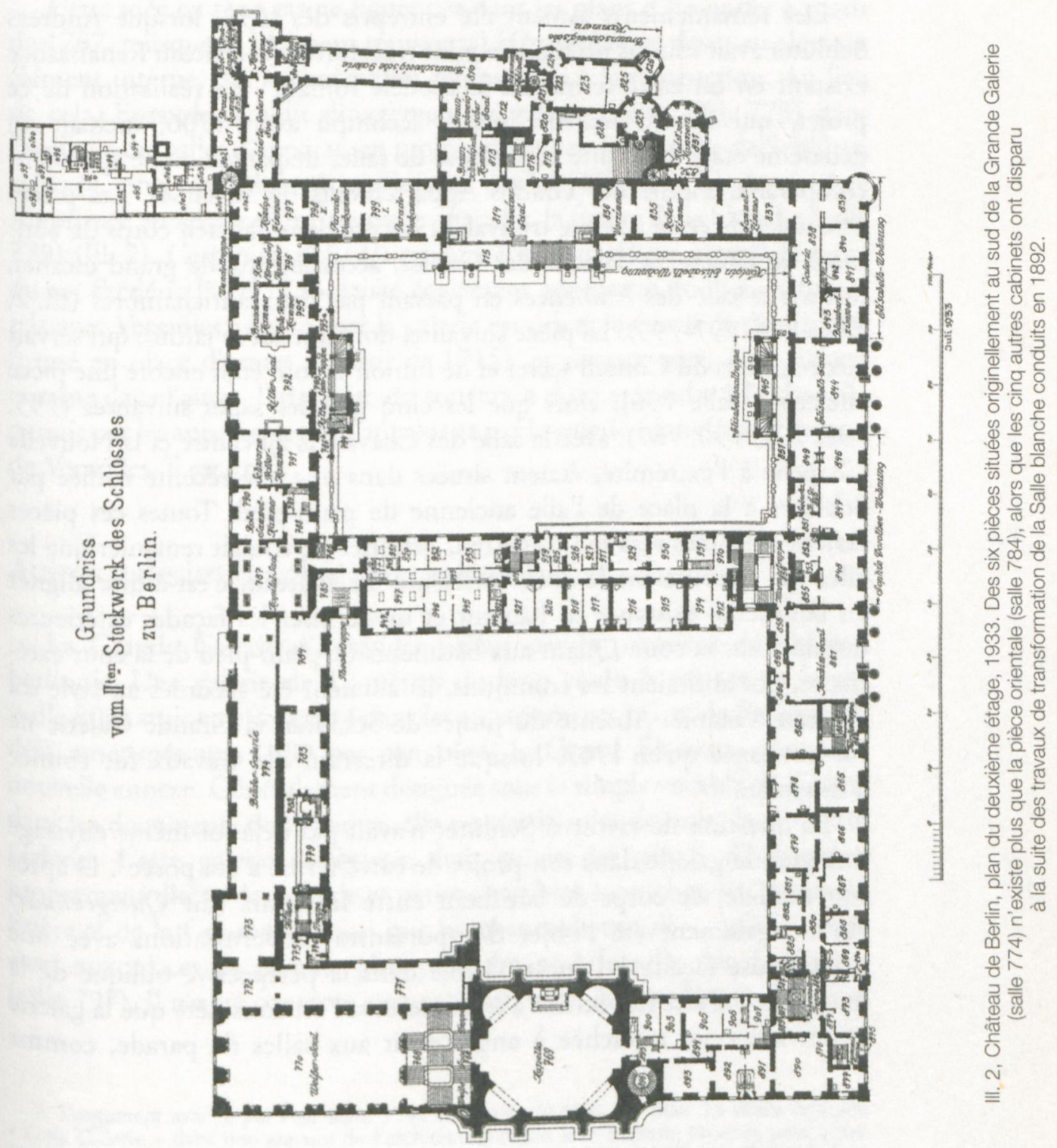




\section{L'édification de la Grande Galerie}

Des remaniements avaient été entrepris dès 1698, lorsque Andreas Schlüter avait soumis un modèle pour transformer le château Renaissance existant en un carré fermé sur le modèle romain ${ }^{4}$. La réalisation de ce projet, qui fut en majeure partie accompli avant 1706, incluait au deuxième étage une suite ambitieuse de salles de parade d'une splendeur comparable à celle des Grands Appartements de Versailles. Les pièces orientales de cette suite se trouvaient encore dans l'ancien corps de bâtiment et allaient de la salle des Gardes, accessible par le grand escalier, jusqu'à la salle des Audiences en passant par deux antichambres (Ill. 2, salles $814,797-799)$. La pièce suivante, donnant sur le jardin, qui servait aux réunions du Conseil secret et de fumoir le soir, était encore une pièce ancienne (salle 796), alors que les cinq grandes salles suivantes (795, $792,791,790,787)$, avec la salle des Chevaliers au centre et la Nouvelle Chapelle à l'extrémité, étaient situées dans une aile récente édifiée par Schlüter à la place de l'aile ancienne de plain-pied. Toutes ces pièces étaient orientées vers le Lustgarten. Schlüter ne voulait remanier que les ailes qui entouraient la cour intérieure du château, c'est-à-dire aligner les bâtiments existants en hauteur et moderniser les façades extérieures donnant sur la cour. Quant aux bâtiments de plain-pied de la cour extérieure, qui abritaient les communs, ils auraient été accordés au style du château remanié. Absente du projet de Schlüter, la Grande Galerie ne fut envisagée qu'en 1707 , lorsque la direction des travaux fut confiée à Eosander.

La question de savoir si Schlüter n'avait pas déjà lui-même envisagé une grande galerie dans son projet de carré fermé a été posée 5 . D'après son modèle, ce corps de bâtiment entre les cours (dit Quergebäude) aurait également été l'objet d'importantes modernisations avec une prestigieuse façade à l'ouest, visible dans la perspective oblique de la maquette $^{6}$. Cette hypothèse a son intérêt si l'on considère que la galerie aurait alors été rattachée à angle droit aux salles de parade, comme

\footnotetext{
4. Hinterkeuser, 2003.

5. Klünner, Peschken, 1982 : 485; Peschken, 1992 : 132-135.

6. Hinterkeuser, 2003 : cat. $n^{\circ} 107$ et 122.
} 
l'était la galerie des Glaces du château de Versailles. Rien cependant ne permet d'attester une telle galerie sous Schlüter ${ }^{7}$.

Cette idée en tout cas ne figure pas dans les plans d'Eosander à partir de 1707, puisque ce bâtiment transversal (Quergebäude) devenait alors un élément interne, qui fut même un temps voué à la destruction. Au lieu de cela, Eosander établit directement sa galerie (salles 774, 773) dans l'enfilade des salles d'apparat, en prolongement de la chapelle de Schlüter, achevée cinq ans plus tôt seulement (salle 787), et qui devait être supprimée en faveur d'une nouvelle chapelle, la future Salle blanche (salle 770) (Ill. 2). Ce projet, dont les travaux furent conduits jusqu'à la mort du roi Frédéric I ${ }^{\text {er }}$ en 1713, laisse également apparaître des liens structurels avec Versailles : d'une part la galerie est orientée vers le jardin (transformé en place d'armes à partir de 1715), et d'autre part, dans un cas comme dans l'autre, l'itinéraire du souverain pour se rendre à la chapelle passait par les appartements d'apparat et par la galerie (dans le sens inverse de Versailles, il est vrai).

\section{Autres galeries du château de Berlin}

La Grande Galerie d'Eosander n'était pas la première du château berlinois. Une galerie de 19 mètres de long et de 5 mètres de large ${ }^{8}$ (salle 800) avait en effet existé dans les appartements privés de Frédéric I ${ }^{\text {er }}$, déjà aménagés vers 1680 par son père, le Grand Électeur, dans une nouvelle annexe. Généralement désignée sous le simple vocable de Galerie dans les documents de l'époque, elle est par la suite devenue la Boisierte Galerie. Cette galerie lambrissée était suivie des pièces d'habitation proprement dites - les grande et petite chambres à coucher, le cabinet de Prière et de la Couronne, ainsi que le cabinet de travail -, qu'elle reliait aussi aux pièces de parade, plus précisément à la salle des Audiences (salle 797). Il n'a été conservé aucune illustration de son décor original et

7. L'argument avancé par Peschken, 1992 (voir note 5) n'est pas clair. La pièce intitulée "G.de Galerie " dans une gravure de l'architecte français Jean-Baptiste Broebes peut s'être trouvée à I'entrée du bâtiment transversal, mais plus vraisemblablement à l'emplacement de la galerie effectivement réalisée par Eosander.

8. Geyer, 1936, vol. texte : 68 ; Klünner, Peschken, $1982: 500$ sq. 
les photos aujourd'hui conservées montrent un lambris moderne en bois de chêne; il existe cependant une documentation concernant un plafond de stuc richement ouvragé. On peut supposer que cette salle servit initialement de galerie de tableaux, mais aucune source ne permet de l'affirmer clairement.

À proximité immédiate se trouvait une deuxième galerie créée quelques années plus tard seulement, entre 1685 et 1690 . Désignée sous le nom de Braunschweigische Galerie9 (galerie de Brunswick, salle 810), celle-ci était un peu plus étroite que la Boisierte Galerie, mais d'une longueur bien supérieure avec ses $35 \mathrm{~m}$. Elle avait pour fonction de relier l'appartement privé du prince électeur et roi aux appartements privés de son épouse situés dans un corps de bâtiment de l'ancien édifice Renaissance. On peut voir dans cette fonction de lien entre les appartements privés du couple régnant une analogie avec Versailles, où la galerie des Glaces reliait également les appartements du roi et de la reine. Mais la ressemblance s'arrête là, son intégration hétérogène dans le plan du château, par exemple, étant bien différente. L'architecte, Johann Arnold Nering, la conçut comme une construction parfaitement autonome semblable à un viaduc, avec des arcades ouvertes dans les deux niveaux inférieurs. Cet exemple montre le rôle et le sens conférés à la fin du XVII ${ }^{e}$ siècle dans le Brandebourg et la Prusse à ces galeries envisagées comme des passages pour relier un édifice existant à un nouveau corps de bâtiment. Cette idée d'architecture additive était étrangère à Schlüter, et son projet de château indique qu'il voulait y mettre un terme ${ }^{10}$. Mais il ne put mener à bien son idée, ce qui ne surprendra personne, puisque ni le roi ni la reine ne souhaitaient une remise en chantier de leurs appartements. La Braunschweigische Galerie semble n'avoir eu qu'une fonction privée, ce que révèle aussi l'iconographie de la peinture du plafond qui avait pour thème l'Amour et Psyché. Bien qu'elle ait été ornée d'un riche décor de stuc et de peinture, on ignore si cette galerie a jamais été requise pour de grandes réceptions ou des fêtes du château. 


\section{Forme et décoration de la Grande Galerie}

C'est précisément le rôle qu'allait jouer à l'avenir la Grande Galerie d'Eosander qui était directement raccordée aux pièces officielles. D'une longueur de $60 \mathrm{~m}$ sur 7,50 m de large et 9,40 m de haut, elle surpassait déjà dans ses dimensions les deux galeries précédentes. Les contraintes du cahier des charges rendaient son implantation complexe. Mais Eosander fut libre de l'intégrer dans le contexte d'un appartement double, c'est-àdire avec une suite de pièces plus petites à l'arrière, alors que les salles d'apparat de Schlüter répondaient au type de l'appartement simple. À cela s'ajouta l'idée de couvrir la salle d'un plafond voûté, et de doter les deux extrémités de paires de colonnes derrière lesquelles s'ouvriraient des pièces attenantes dont on remarque que l'orientale était beaucoup plus courte que son pendant occidental et ne disposait que d'un seul axe de fenêtres.

Une coupe moderne (Ill. 3) de cette pièce attenante orientale fait apparaître, si l'on s'arrête sur la rupture abrupte de la voûte, qu'Eosander avait peut-être envisagé de supprimer ultérieurement toute l'ancienne chapelle afin de l'intégrer partiellement ou même complètement à cette pièce orientale de la galerie ${ }^{11}$. Le raccord de cette aile à celle qui avait été précédemment édifiée par Schlüter a en tout cas présenté des difficultés, la création de la pièce plus courte l'obligeant d'emblée à empiéter sur l'ancienne chapelle et à la priver d'un axe de colonnes. L'autorisation ne lui fut pas accordée de détruire entièrement la chapelle, ce qui aurait du reste été très coûteux en raison de la complexité de la voûte.

Mais pourquoi Eosander avait-il besoin dès cette période de l'axe de la chapelle? N'aurait-il pu se contenter de raccorder simplement sa galerie à la chapelle? Cette question appelle une réponse négative si l'on observe la façade extérieure. Eosander était en effet contraint de prévoir, en prolongeant l'aile à cet endroit, un autre portail, le portail IV, dont l'emplacement était prescrit par le portail situé à l'opposé sur la place du Château (Schlossplatz). S'il avait tout simplement raccordé à la chapelle cette pièce contiguë de la galerie, elle se serait trouvée tout entière derrière ce portail, et donc exclusivement éclairée par de très grandes fenêtres, ce qui ne convenait pas. L'unique fenêtre de la pièce attenante orientale est

11. Geyer, 1992, vol. illustrations : ill. $\mathrm{n}^{\circ} 28$. 
en effet beaucoup plus large que les autres fenêtres du château, comme il apparaît clairement dans le plan en coupe (Ill. 3). La dimension des fenêtres était a priori prescrite par le système de façade de Schlüter et concernait aussi bien les parties arrière que les portails. Contraint d'en tenir compte, Eosander ne pouvait guère se permettre d'ouvrir de grandes arcades dans la galerie à l'instar de Versailles.
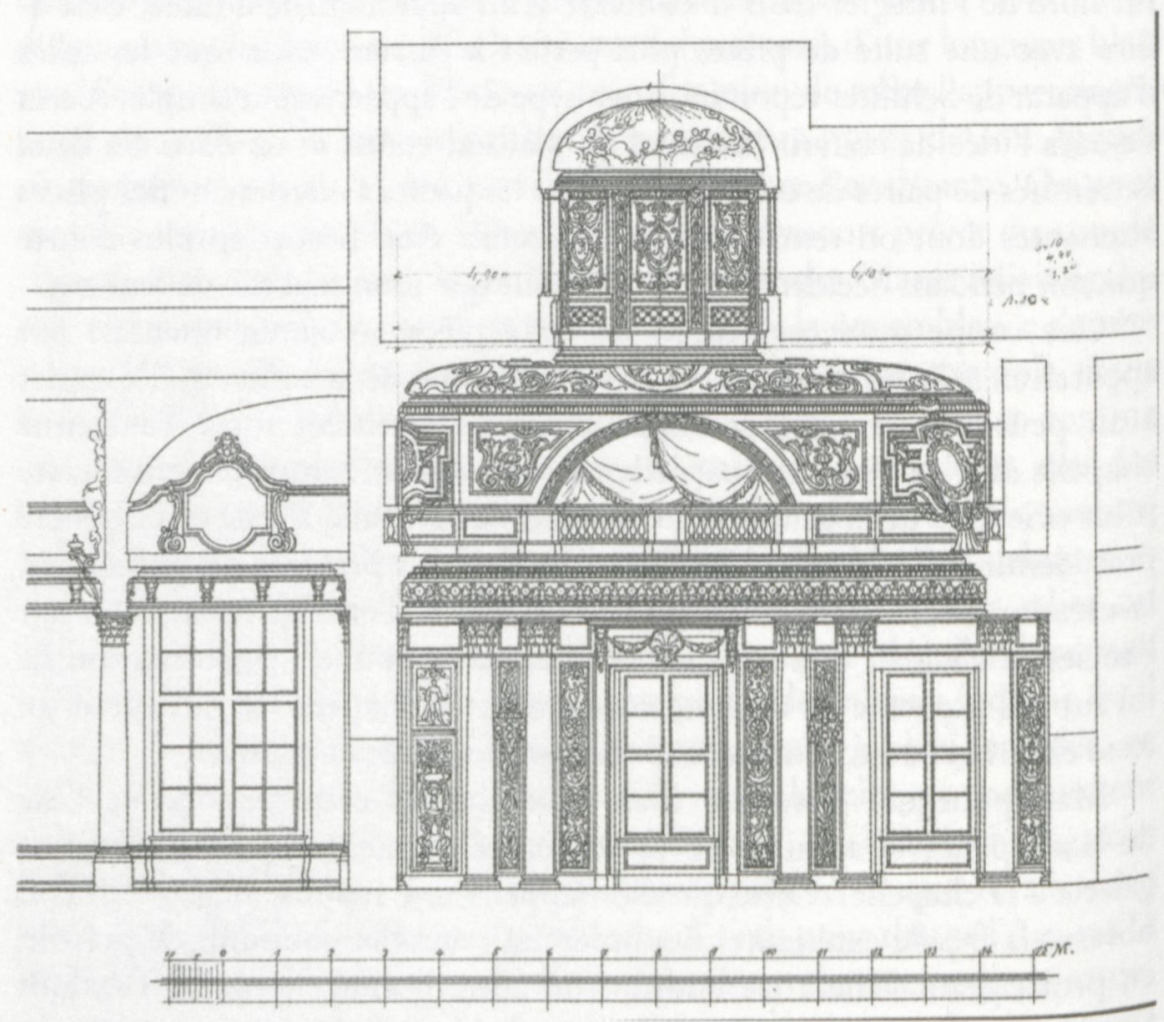

III. 3, Albert Geyer, coupe de l'extrémité orientale de la Grande Galerie du château de Berlin avec la pièce orientale et, à droite, l'ancienne chapelle adjacente.

Aux huit fenêtres que comptait la galerie proprement dite furent ajoutées une fenêtre dans la pièce attenante orientale et deux dans la pièce attenante occidentale. Même si le décor originairement prévu ne fut pas achevé, on doit constater que les dépenses pour les coûteux matériaux, 
en particulier le marbre, furent d'emblée très réduites : seules les deux colonnes du côté occidental étaient de marbre, ainsi que quelques pilastres dans les cabinets; les colonnes orientales, parce qu'elles se trouvaient directement au-dessus du porche et ne pouvaient par conséquent excéder un certain poids, furent réalisées en marbre stuqué, les chapiteaux des colonnes, comme les éléments plastiques du plafond le furent en stuc. Les ouvrages en stuc, réalisés par l'un des sculpteurs français actifs à Berlin, peut-être Charles Claude Dubut ${ }^{12}$, constituaient avec les plafonds peints les éléments les plus remarquables du décor.

Au milieu de la galerie, sur la corniche surplombant les deux murs longitudinaux, se trouvaient de chaque côté deux grands groupes figurant les quatre continents : l'Europe et l'Asie sur le mur le mieux éclairé, l'Afrique et l'Amérique du côté des fenêtres (Ill. 1). D'autres groupes allégoriques et de putti s'ajoutaient à l'ensemble de part et d'autre. L'espace situé au-dessus des colonnes, à l'est comme à l'ouest, était lui aussi couvert de grands reliefs, groupes allégoriques illustrant le règne salutaire du maître d'ouvrage, Frédéric I I : à l'est, l'essor des arts et des sciences refoulant les vices (IIl. 4), à l'ouest, une allégorie du couronnement et du serment de fidélité de Frédéric ${ }^{\text {Ir. }}$. À la différence du décor de la galerie des Glaces de Versailles, les thèmes iconographiques étaient exclusivement centrés sur des faits intervenus à l'intérieur du royaume, sans aucune mention des succès guerriers et de politique extérieure.

Cela valait aussi pour la peinture du plafond. Mais seules les quatre peintures des lunettes latérales qui surmontaient la corniche et se rejoignent par deux au sommet étaient de l'époque de Frédéric Ir (Ill. 1). Trois d'entre elles, du peintre flamand Antoine de Coxcie, le figuraient en juge, en législateur ou en protecteur de la religion. La quatrième représentait le couronnement de son épouse Sophie Charlotte par lui-même. Selon la conception initiale, trois peintures centrales étaient prévues pour ce plafond, structuré par l'encadrement des tableaux, des draperies et des groupes de putti, mais aucune ne fut réalisée. Le dernier état conservé du décor, composé d'un tableau central flanqué de médaillons, date de la rénovation de la galerie commandée par l'empereur Guillaume II au $\mathrm{XX}^{\mathrm{e}}$ siècle. Seule la voûte de la pièce occidentale (salle 773 ) avait été 
entièrement ornée, dès 1713 environ, d'une peinture à l'huile allégorique, du peintre berlinois Johann Friedrich Wentzel probablement, illustrant les vertus du règne prussien, mais d'une qualité artistique médiocre et surchargée d'un enchevêtrement de références iconographiques ${ }^{13}$.

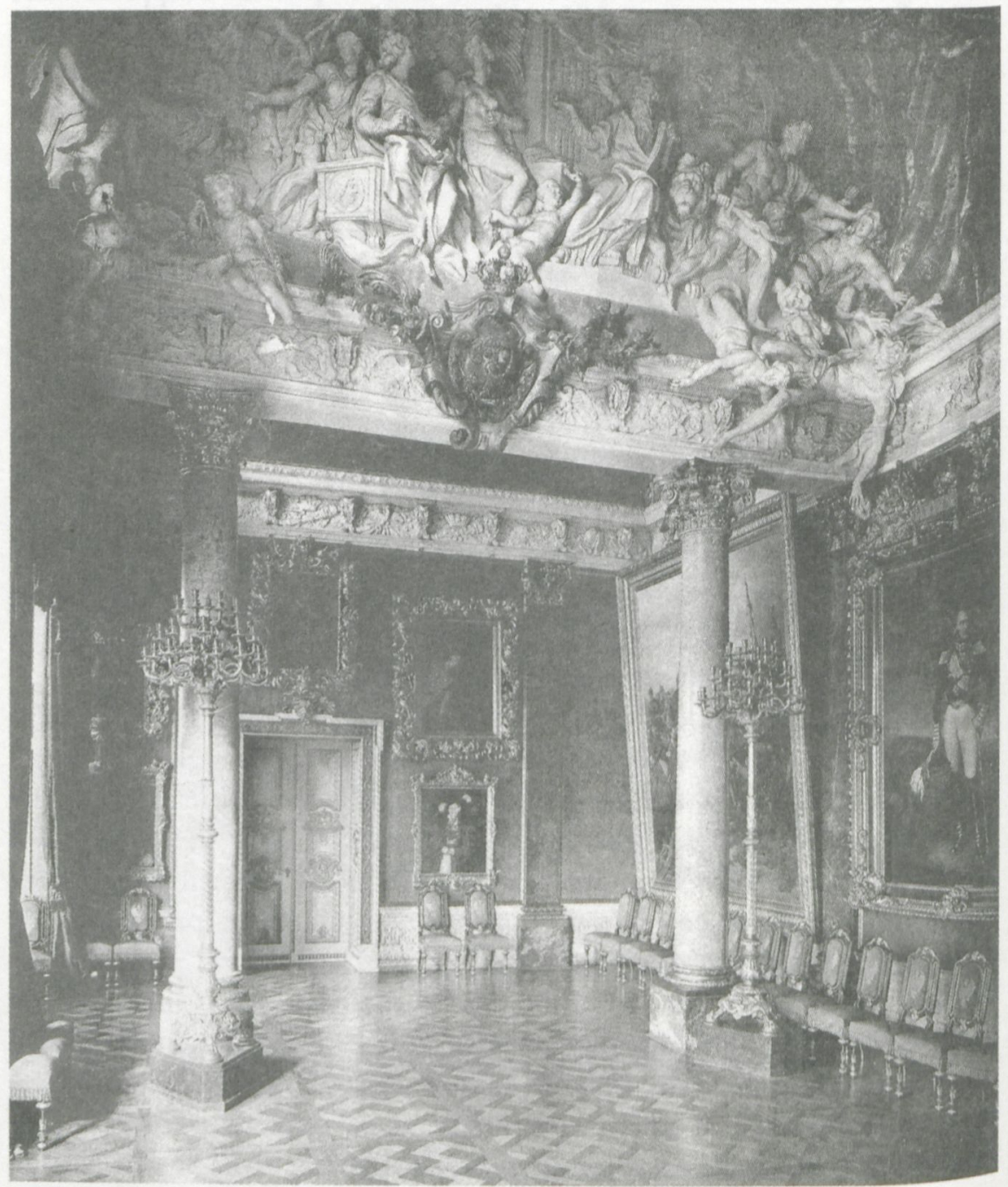

III. 4, Château de Berlin, vue de la pièce attenante orientale vers 1870.

13. Essai d'interprétation dans Wiesinger, 1992 : 190-191. 


\section{Les plans pour l'aménagement sous Frédéric ler}

La galerie étant restée inachevée à la mort de Frédéric I ${ }^{\mathrm{er}}$, en 1713 , seules des suppositions sont permises quant à la décoration intérieure et au mobilier envisagés. Il est néanmoins vraisemblable que des miroirs aient été prévus sur le mur intérieur longitudinal, comme c'est le cas dans les galeries de Versailles et de Stockholm. Par ailleurs, nous savons qu'en 1712-1713 des paiements ont été versés à l'orfevre Andreas Haidt pour des "cadres en laiton doré $[\ldots]$ pour la nouvelle galerie d'art ${ }^{14}$ ". Cette source pourrait indiquer que Frédéric $\mathrm{I}^{\mathrm{er}}$ souhaitait aménager une véritable galerie de peintures, mais il est aussi possible que ces cadres aient servi à encadrer des miroirs. Ces cadres, dont nous ne savons pas ce qu'ils sont devenus, auraient peut-être été placés sur le mur extérieur entre les fenêtres et en vis-à-vis sur le mur intérieur.

Il ne fait aucun doute que c'est le modèle de la galerie des Glaces de Versailles, presque entièrement achevée en 1684, qu'Eosander aura eu en tête lors de sa conception, même s'il dut se restreindre sur de nombreux points. Dès l'année 1700 , il avait été spécialement envoyé à Paris par la reine Sophie Charlotte (1668-1705), afin de se familiariser avec les décors intérieurs des châteaux français. Dans ses missions d'études, il reçut même le précieux soutien de la duchesse Élisabeth Charlotte de Bavière, duchesse d'Orléans (1652-1722). Les intérieurs réalisés par Eosander à son retour à Berlin, notamment dans le château de Charlottenburg, témoignent du reste de fortes influences françaises ${ }^{15}$.

Mais, plus encore que la galerie des Glaces de Versailles, c'est le modèle de la galerie du château de Stockholm que l'on reconnaît ici ${ }^{16}$. Créée en 1693 sur les plans de Nicodème Tessin le Jeune, elle avait été très gravement endommagée en 1697 dans le grand incendie du château, puis reconstruite les années suivantes. Son décor de miroirs, de stuc et

14. Mémorial de Johann Friedrich Eosander : "Dem Goldschmiede Andrea Haydt für verfertigte Messinge übergüldete Schilderey Rähme 688 thlr. - dito demselben für dergleichen verfertigte Schilderey-Rähme 722 thlr. - noch dem Gold Schmiede Haydt für dergleichen Schilderey-Rähme zu der neuen Kunst Gallerie 813 thlr. », cité dans Ladendorf, 1935: 166, note 79.

15. Hinterkeuser, 2005-2006 : 254; Hinterkeuser (à paraître).

16. Voir à ce sujet I'article de Martin Olin dans le présent ouvrage. 
de peintures rappelle le modèle versaillais. On sait que Tessin engagea surtout des artistes français pour l'aménagement des intérieurs du château suédois. Eosander connaissait personnellement Tessin et il lui rendit même visite en 1704 à Stockholm, où il put voir la galerie alors presque entièrement reconstruite. C'est à cette occasion qu'Eosander prit connaissance des plus récentes tendances modernes de l'art français, si bien que certaines idées venues de France pourraient être arrivées à Berlin via Stockholm ${ }^{17}$. À l'époque où il s'y trouvait, les plans du projet de galerie prévoyaient du reste encore une séparation des deux salons mitoyens par des couples de colonnes, comme à Berlin ${ }^{18}$. Des années plus tard seulement, Tessin renonça à ce plan imposant et, lors de la réalisation, il remplaça les colonnes par des consoles ${ }^{19}$. Mais dans son Traictè de la décoration intérieure de 1717 , la solution des colonnes dégagées semble encore retenue ${ }^{20}$. Il ne fait pas de doute que Tessin s'est ici inspiré de la galerie Colonna (H-T. 7) qu'il connaissait pour l'avoir vue personnellement ${ }^{21}$. Mais la part d'influence de l'exemple romain n'intervient qu'indirectement à Berlin, avec ce détour par Stockholm précisément. Le plan de Stockholm suffit entièrement à expliquer la conception d'Eosander pour Berlin, puisqu'il l'a vu de ses yeux, alors que nous ignorons s'il est jamais allé en Italie.

La galerie n'ayant pas été achevée sous Frédéric $\mathrm{I}^{\mathrm{er}}$, il est difficile de déterminer le rôle qui lui était affecté à l'intérieur du cérémonial de cour. Mais cet aspect de passage, d'itinéraire de parade pour se rendre à la chapelle, a certainement prévalu. On ignore s'il s'agit là d'une persistance de la tradition entretenue depuis le XVI ${ }^{\mathrm{e}}$ siècle, où le cercle des proches de la cour se rendait au dôme par les toits plats des édifices qui bordaient les deux cours ${ }^{22}$. L'idée d'un passage couvert apparaît déjà à cet endroit dans

17. Hinterkeuser, $2001: 266$.

18. Kommer, 1974 : pl. $28,34,40$.

19. Ibid. : cat. $\mathrm{n}^{\circ} 42$.

20. « L'Ornement et les Chapiteaux sont dorès par tout, et les bases avec leurs zocles sont de bronze d'orè, et de mesme tous les ornemens, qui sont faits à jour sur les Pillastres et Colonnes. Entre chaque croisee il y a deux de ces Pillastres de marbre blanc, et à chacque bout vers les deux Cabinets, deux Colonnes entieres et deux demies. " Cité dans Kommer, $1974: 165$.

21. Strunck, $2007: 368-369$.

22. Geyer, $1936: 50$ sq. 
la maquette du château de Schlüter ${ }^{23}$. Dans le cérémonial diplomatique de cour proprement dit, la galerie n'aurait certainement pas été prescrite. La mission des légations prenait fin dès la salle des Audiences, les salles officielles mitoyennes, avec la salle des Chevaliers au centre, n'étant utilisées dans la mesure du possible que pour quelques célébrations de cour annuelles, dont la fête de la fondation du Schwarzer Adlerorden (Ordre de l'Aigle noir). Pour les festivités et les bals, également prévus au programme des visites d'État, les suites étaient ouvertes et solennellement éclairées. La galerie se serait parfaitement insérée dans cette enfilade.

\section{L'aménagement sous Frédéric Guillaume ${ }^{\text {er }}$ : de la galerie d'Argent à la galerie de Peintures}

Lorsque Frédéric Guillaume I ${ }^{\mathrm{er}}$, fils de Frédéric $\mathrm{I}^{\mathrm{er}}$, accéda à la régence en 1713 , il poursuivit le projet d'agrandissement de son père, après une brève interruption, et l'acheva en 1716. Il apporta néanmoins quelques innovations dans les salles d'apparat. Il commença par ordonner l'interruption immédiate de la construction de la nouvelle chapelle, ce qui lui valut quelques procès avec des sculpteurs hollandais pour refus de réception et paiement de colonnes de marbre préalablement commandées ${ }^{24}$. La galerie devenait alors le point culminant des pièces d'apparat à l'ouest. Il la déclara par ailleurs provisoirement terminée en ordonnant l'arrêt des travaux aux peintures des plafonds, exigeant même que murs et plafonds, dont les peintures déjà réalisées, soient intégralement recouverts d'une couche de blanc afin de conférer à la galerie à demi achevée " une apparence égale ${ }^{25}$ ". Les lambris de chêne des murs, mentionnés pour la première fois en 1794, furent sans doute aussi réalisés sous son égide ${ }^{26}$. Enfin, il mit spectaculairement en scène la galerie en la garnissant d'un riche mobilier d'argent, à savoir dix grands miroirs et près de deux cents bras de lumière. Ces miroirs et appliques d'éclairage

23. Hinterkeuser, 2003 : cat. $\mathrm{n}^{\mathrm{os}} 107$ et 122.

24. Geyer, 1992, vol. texte : 30.

25. "[...J ein egales Ansehn », Schasler, 1856: 173.

26. "Sie [die Galerie] ist durchaus mit eichenem Holz getäfelt, mit goldenen Leisten eingefaßt, und mit Perlenfarbe angestrichen. " (La galerie est " lambrissée de bois de chêne avec des baguettes dorées, et peinte couleur blanc perle ») Rumpf, 1794 : 79. 
ne constituent cependant pas un ensemble homogène dans leurs formes ni dans leurs dimensions - les éclairages en particulier sont de types très variés -, puisque non spécialement créés pour la galerie, mais provenant d'autres châteaux. Deux inventaires de l'argenterie datés des années 1713 et 1715 en font respectivement mention en détail ${ }^{27}$. Un miroir ${ }^{28}$ acquis en l'an 2000 par la Fondation des châteaux et jardins prussiens de Berlin Brandebourg - sans doute originellement accroché dans la salle à manger du roi (Tafel-Saal des Königs) - permet d'imaginer l'aspect de ceux qui furent accrochés dans la galerie.

L'argent étincelant sur les murs blancs aura eu un effet singulier. Mais comment expliquer cette sobriété d'une part et le coût de ce somptueux mobilier d'argent de l'autre? L'explication est simple. Peu de temps après sa prise de pouvoir en 1713, Frédéric Guillaume I ${ }^{\text {er }}$ ordonna que l'ensemble de l'argenterie du château de Charlottenburg et des autres palais de campagne de son père fût enlevé et rassemblé dans les salles d'apparat du château de Berlin. La galerie fut donc elle aussi abondamment garnie d'argent. Frédéric Guillaume I ${ }^{\text {er }}$ aura été conscient du puissant effet produit par un mobilier d'argent, tellement supérieur à celui de toutes peintures murales, a fortiori si l'on considère la médiocrité des peintres berlinois. D'abord tenté d'ordonner la fonte du "précieux ouvrage d'argent ${ }^{29}$ ", il avait fini par se laisser convaincre que "ce serait grand dommage en raison de la rareté de l'ouvrage ${ }^{30}$ ".

Tel a finalement été le bon plaisir de Votre très gracieuse Majesté, afin d'ajouter à la magnificence du château, d'en ordonner la répartition dans les différentes chambres et ainsi les ornementer plus que royalement [...] Chacun en admire le prodigieux $\operatorname{trésor}^{31}[\ldots]$

27. Das Silber in den Paradekammern und Kammern Friedrich I. Majst. im Schlosse zu Berlin Anno 1713, p. 34-39 (Potsdam, Stiftung Preußische Schlösser und Gärten BerlinBrandenburg, Plankammer, inventaire $n^{\circ} 26$ ). Zusammenstellung der sämtlichen Manual des Koenigl. Silbers von Trinit. 1715 Biss Trinit. 1716 (Potsdam, Stiftung Preußische Schlösser und Gärten Berlin-Brandenburg, Plankammer, inventaire ${ }^{\circ}{ }^{\circ} 27$ ), cité dans Seidel, 1896: 9-11.

28. Arminjon, 2007 : cat. $n^{\circ} 90$.

29. "[...] pretieuse Silberwerck ", Berliner geschriebene Zeitung, 1902, 3 juin 1713 : 13-14.

30. "[...] das es wegen der raren façons großer Schade seyn würde ", ibid. : 14.

31. "I...] haben Sie endlich allergn. beliebet, es zum mehreren Lustre des Schloßes in denen Gemächern zu vertheilen und sie damit mehr als Königlich zieren zu laßen [...] Ein jeder admiriret den ungemeinen Schatz [...] ", ibid. : 14. Concernant le mobilier d'argent actuel du château berlinois sous les deux premiers rois de Prusse, on se reportera à Baer, 2007 : 125-141. 
Le mobilier d'argent de la cour berlinoise échappa donc en 1713 au sort réservé à celui de Versailles sur ordre de Louis XIV dès la fin de l'année $1689^{32}$.

On peut voir dans ce décor de miroirs d'argent une contradiction avec les idées de Frédéric I ${ }^{\text {er }}$, et parler ici d'une solution fortuite. Car il n'aurait personnellement pas disposé de telles quantités d'argent pour le château de Berlin, n'ayant pas eu l'intention de vider ses autres châteaux. Par ailleurs, malgré l'opulence affichée de ce mobilier, le décor avait quelque chose de désuet. À la place de ces miroirs individuels essentiellement mobiliers, il aurait été plus hardi de créer des miroirs muraux directement intégrés à la décoration d'ensemble. C'était déjà une pratique très habituelle alors dans les châteaux français, et cette mode avait aussi gagné Berlin depuis longtemps, comme en témoignent différentes pièces du château de Charlottenburg. Dans la galerie des Glaces de Versailles comme dans celle du château de Stockholm, le long mur opposé aux fenêtres est ainsi garni de miroirs. Eosander avait sans doute envisagé une telle solution pour sa galerie, mais la mort de Frédéric I ${ }^{\mathrm{er}}$ en empêcha la réalisation.

Cet exubérant décor de miroirs et bras de lumière aura presque complètement recouvert les murs, ce qui exclut l'hypothèse de la présence d'une collection de tableaux dans la galerie en 1713. C'est quelques années plus tard seulement que des peintures sur toile y furent introduites. La date exacte de ce nouveau remaniement n'est pas connue, mais il a vraisemblablement eu lieu avant l'année 1728, lorsque Auguste le Fort, électeur de Saxe et roi de Pologne, visita le château berlinois. Les salles d'apparat furent alors somptueusement éclairées, comme il ressort d'un rapport spécifique sur les dépenses enregistrées pour les bougies de cire ${ }^{33}$. La suite des pièces, généreusement illuminées pour la circonstance, commençait dans la salle Suisse et se terminait "dans la Grande Galerie" ( in der großen Gallerie "), dans laquelle se trouvaient, outre les 104 doubles chandeliers "placés sous les corniches " ( unter denen Gesimbsen »), 9 grands lustres en couronnes et 4 petits, ainsi que 20 guéridons. Ce qui signifie

32. Mabille, 2007: 77-82 [voir aussi note 36].

33. "Specification Was die weißen wachßlichter welche in denen Königl. Gemächer aufgestecktem an gelde betragen ", in Acta betr. den Aufenthalt des Königs von Polen in Berlin und Potsdam im lahre 1728, p. 10 (Berlin, Geheimes Staatsarchiv Preußischer Kulturbesitz, I. HA, Rep. 36, n² 2950/1). 
que les 200 bras de lumière - de même que les miroirs d'argent probablement - mentionnés en 1713 et 1715 ne s'y trouvaient pas. Leur absence ne peut s'expliquer que par le besoin de libérer les murs pour l'accrochage de tableaux. En 1742, dans un inventaire sommaire établi deux ans après la mort de Frédéric Guillaume I ${ }^{\mathrm{er}}$, le relevé de son trésor d'argent fait état pour la galerie de " 10 lustres, 24 guéridons $^{34}$ ", l'aménagement de la galerie concernant l'éclairage ayant été peu modifié depuis 1728 . On note par ailleurs que les éléments cités sont expressément enregistrés comme de nouvelles créations commandées par le roi. Le changement des luminaires ne visait par conséquent pas seulement à libérer les surfaces murales pour les tableaux, mais aussi à remplacer le bric-à-brac des éléments anciens par un ensemble homogène et moderne.

D'après l'état actuel des recherches, la présence d'une partie importante de la collection de peintures dans la galerie est explicitement attestée pour la première fois en novembre 1731 à l'occasion du mariage de Wilhelmine de Prusse, sœur de Frédéric le Grand, avec Frédéric III, futur margrave de Brandebourg-Bayreuth, célébré dans les salles d'apparat, incluant la Salle blanche, récemment achevée. Dans ses mémoires, Wilhelmine mentionne explicitement - et en français - la "galerie ornée de très-beaux tableaux ${ }^{35}$ ». Cette information s'accorde avec les sources mentionnées plus haut sur le changement de l'éclairage.

\section{La galerie de Peintures sous Frédéric le Grand et Frédéric-Guillaume II}

Il est donc établi que la conversion en galerie de peintures avait déjà eu lieu sous Frédéric-Guillaume ${ }^{\text {Ir } 36}$. Au cours des années suivantes, elle sera à plusieurs reprises décrite comme telle. Dans un témoignage de 1744, on lit :

La galerie, longue de plus de 50 pas, est ornée des meilleures peintures de la main des célèbres Italiens Titien, Tintoret, Paul de Vérone, Raphaël

34. Cité dans Seidel, $1896: 33$.

35. Wilhelmine, $1967: 203$.

36. Et non sous Frédéric le Grand, qui, en cela un peu semblable à Louis XIV, fit fondre en 1745 la presque totalité des trésors de la plus précieuse argenterie de son palais, y compris les meubles, pour financer ses guerres, voir Seidel, $1896: 36-41$. 
d'Urbin, et du Néerlandais van Dyck, mais les sols sont de bout en bout parquetés ${ }^{37}$.

Et en 1749 est mentionnée « la longue galerie entièrement ornée de précieuses peintures ${ }^{38}$ ", en 1756 " une galerie dans laquelle sont accrochés les portraits les plus rares et les plus précieux ${ }^{39}$ ".

Ce sont ensuite les descriptions de la fin du XVIII ${ }^{\mathrm{e}}$ siècle qui répertorient en détail, tableau après tableau, la totalité de la collection ${ }^{40}$. Elle comptait à parts égales des maîtres néerlandais, italiens et français, mais très peu d'Allemands et aucun peintre prussien. On y trouve les noms de Rubens, Rembrandt, Van Dyck, Jordaens, Titien, Giorgione, Reni, Véronèse, du Corrège, du Caravage, de Watteau, Poussin et enfin d'Antoine Pesne aussi, le peintre de la cour prussienne d'origine française. Si ces attributions ne sont pas toutes attestées aujourd'hui, l'ensemble forme néanmoins l'origine de la présente collection de la Gemäldegalerie berlinoise. Une visite de l'actuelle galerie de peintures de Frédéric le Grand à SansSouci ${ }^{41}$ permet de se faire une idée du décor et de l'accrochage des œuvres dans l'ancienne galerie du château de Berlin.

En 1786, la galerie de tableaux du château de Berlin comptait 251 peintures, auxquelles s'ajoutaient 350 autres dans les 3 cabinets qui la jouxtaient côté jardin et dans les 6 cabinets alignés côté cour, le long du mur intérieur de la galerie ${ }^{42}$. Les œuvres présentées dans ces pièces n'étaient pas non plus classées par écoles, nationalités ou types : paysages, portraits, peintures historiques ou scènes de genre étaient mêlés indistinctement. Après la mort de Frédéric le Grand en 1786, la collection de peintures fut étendue sous cette même forme jusqu'à la salle des Fêtes principale, la Salle blanche mitoyenne, qui n'abritait jusque-là que 11 portraits officiels ${ }^{43}$. En 1794 , l'ensemble des 11 pièces, Salle blanche

37. Schramm, $1744: 147-148$.

38. "[...] die lange mit kostbahrsten Gemählden ausgezierte Gallerie ", Keiser, 1749: 22.

39. " [...] eine Gallerie mit den allerkostbarsten und raresten Portraits behangen ", Müller, Küster, $1756: 18$.

40. Nicolai, $1786: 884-900$.

41. Die Bildergalerie in Sanssouci, 1996.

42. Nicolai, $1786: 884-900$.

43. Nicolai, 1786 : 896; Rumpf, 1794 : 116-128. 
comprise, constituant la galerie, réunissait beaucoup plus de 900 peintures - au lieu des 600 tableaux à peine en 1786. Dans la galerie proprement dite, le nombre de tableaux avait au contraire été réduit de 251 à 104. Il s'y trouvait par ailleurs 7 grandes tables garnies de somptueux plateaux de marbre sur lesquels étaient présentés des bustes ${ }^{44}$. L'éclairage de la galerie fut également fréquemment modifié. En 1786, il était fourni par " neuf hauts lustres de bois argenté " ("neun hohe Leuchter von Holz und versilbert ${ }^{45}$ "), ce qui laisse supposer que les guéridons d'argent véritable mentionnés en 1728 et 1742 avaient disparu dans la fonte de $1745^{46}$. En 1794 étaient en revanche venus s'ajouter 10 lustres de cristal $^{47}$, qui ont cependant disparu de nouveau dans les illustrations du XIX ${ }^{\mathrm{e}}$ siècle.

\section{La nouvelle conception de la galerie de Peintures au XIX ${ }^{\mathrm{e}}$ siècle}

En 1830 fut inauguré le musée Royal aménagé dans le bâtiment de Karl Friedrich Schinkel, l'actuel Altes Museum, situé sur la place du Lustgarten. En 1829, cette galerie de peintures nouvellement conçue fut encore dotée de 347 tableaux provenant des différents châteaux royaux, dont 157 prélevés dans la seule galerie du château de Berlin et ses pièces avoisinantes ${ }^{48}$. Les manques qui en résultèrent obligèrent à une conception nouvelle et radicale, accompagnée d'une rénovation des lieux. Le caractère d'ensemble de la galerie ayant été jusque-là plutôt universel, on inclinait maintenant à un retour à l'histoire locale, à la fois du lieu luimême, mais plus généralement aussi de l'État prussien. Dans une restauration conduite en 1835 , la peinture à la chaux du plafond fut enlevée,

44. Rumpf, 1794 : 79-144. La restauration de la galerie, mentionnée en 1791, est probablement aussi consécutive à la réorganisation de I'accrochage des peintures. On ignore la nature exacte des mesures alors entreprises. Voir Borrmann, $1893: 288$.

45. Nicolai, $1786: 884$.

46. Seidel, $1896: 36-46$.

47. Rumpf, $1794: 81$.

48. Vogtherr, $2005:$ 82-93. En 1806 déjà, Vivant Denon avait dérobé quelques-unes des meilleures peintures de la Grande Galerie du château de Berlin, qui furent toutefois restituées après la défaite de Napoléon. Voir Verzeichniß von Gemälde, 1815. 
découvrant les quatre peintures de grand format de Coxcie $^{49}$ avec les portraits de Frédéric ${ }^{\text {er }}$. Le reste de la surface de ce plafond fut peint en bleu, et les murs tendus d'étoffe rouge (H.-T. 14, 15). Les grisailles du peintre Karl Stürmer, scènes du passé brandebourgeois ${ }^{50}$ peintes en rouge sur fond rouge sur des plaques de cuivre dans les espaces encadrés face aux fenêtres des mezzanines, furent aussi une innovation qui renvoyait à l'origine baroque de la galerie. Elles remplaçaient en quelque sorte en trompe-l'œil les reliefs originellement envisagés à cet endroit et dont un seul, figurant la remise de l'Ordre de l'Aigle noir, avait été réalisé à l'extrémité orientale de la galerie ${ }^{51}$.

À la différence du premier accrochage, la collection de peintures de la galerie proprement dite fut alors restreinte à des œuvres pour la plupart contemporaines ${ }^{52}$. Quelques maitres anciens furent accrochés sur les murs transversaux de la galerie, et l'on transféra ceux qui n'étaient pas destinés au musée dans des cabinets voisins où ils furent alors classés par écoles et nationalités des artistes. Pour la première fois, deux cabinets hollandais et deux cabinets italiens voisinaient avec une remarquable section d'anciens maîtres allemands ${ }^{53}$, d'où le nom de Altdeutsche Kammern, adopté pour désigner l'ensemble de ces cabinets ${ }^{54}$.

Sur les murs longitudinaux de la galerie étaient présentés des tableaux illustrant l'histoire de la Prusse et du Brandebourg, complétés par des portraits des souverains régnants. Les protagonistes de la coalition antinapoléonienne, le tsar Alexandre ${ }^{\text {er }}$ de Russie, l'empereur François I ${ }^{\text {er }}$ d'Autriche et le roi de Prusse Frédéric Guillaume III, y étaient présents, ainsi que Napoléon lui-même, visible dans une version du tableau de Jacques Louis David, Bonaparte au Grand Saint-Bernard 55 (H.-T. 15). Avec 60 peintures environ, le nombre d'œuvres s'était encore notablement

49. Schasler, $1856: 172-173$.

50. Geyer, 1992, vol. texte : 17.

51. Schasler, 1856:173; Geyer, 1992, vol. texte : 17. Nous ignorons sur quels documents s'est basé Peschken pour attribuer le relief à Johann Gottfried Schadow. Voir Klünner, Peschken, 1982 : 486; Peschken, Wiesinger, 2001, vol. texte : 199.

52. Schasler, $1856: 174-177$.

53. Ibid., $1856: 189-191$.

54. Morin, $1860: 178$.

55. Schasler, $1856: 175$. 
réduit par rapport à 1794 et rassemblait surtout une suite de formats beaucoup plus grands. Ainsi, la galerie s'éloignait de plus en plus de son caractère baroque d'origine; pour la référence à Versailles, on peut même dire que, délibérément ou non, elle se rapprochait plus, avec ses nombreuses peintures historiques, de la galerie des Batailles, édifiée en 1837, que de la galerie des Glaces.

C'est à partir de cette date marquant une ouverture au présent que des événements nouveaux de l'histoire contemporaine, alors admis comme sujets picturaux, se succédèrent dans la galerie, inaugurant un va-et-vient constant de nouveaux accrochages. Après 1843, la peinture de grand format de Franz Krüger, la Fête en l'honneur du serment de fidélité de Frédéric Guillaume IV à Berlin le 15 octobre 1840, offerte par les citoyens berlinois, fit son entrée dans la galerie ${ }^{56}$ (H.-T. 14, $4^{\mathrm{e}}$ grand tableau sur le mur à gauche). Après 1865 vint s'y ajouter le tableau d'Adolph Menzel, Le couronnement du roi Guillaume Ir à Königsberg ${ }^{57}$. En 1877, pour le quatre-vingtième anniversaire de l'empereur, les princes allemands et les villes autonomes offrirent à Guillaume Ir ${ }^{\text {er }}$ le tableau d'Anton von Werner, La proclamation de l'empereur allemand dans la galerie des Glaces de Versailles ${ }^{58}$, qui fut d'abord accroché dans la pièce attenante occidentale ${ }^{59}$. C'est ainsi qu'une vue de la galerie des Glaces de Versailles fit son entrée dans la Grande Galerie de Berlin - et que le modèle fut montré dans sa réplique. Mais rares seront les visiteurs qui auront considéré le tableau sous cet aspect architectural et historique. En 1893, Anton von Werner achevait sa peinture monumentale, L'inauguration du Reichstag dans la Salle blanche du château berlinois le 25 juin 1888. C'est peut-être ce qui a suscité chez l'empereur Guillaume II l'idée d'un remaniement complet de l'accrochage ${ }^{60}$. On décrocha alors La fête en l'honneur $d u$ serment de fidélité de l'empereur de Krüger; Le couronnement de Menzel et La proclamation de Werner furent décalés vers l'est pour constituer dès lors les points forts de la galerie proprement dite (IIl. 5), alors que L'inauguration

56. Reinhard, $1852: 46$.

57. Baedeker, $1878: 24$. 903),

58. Gaehtgens, 1990; Bartmann, 1993 : 332-349; Bartoschek, Vogtherr, 2004 : 580 (GK I

59. Baedeker, $1887: 70-71$.

60. Borrmann, $1893: 289$. 
$d u$ Reichstag de Werner fut transférée dans la pièce attenante occidentale ${ }^{61}$. C'est aussi à ce moment-là sans doute que fut décroché le Bonaparte de David.

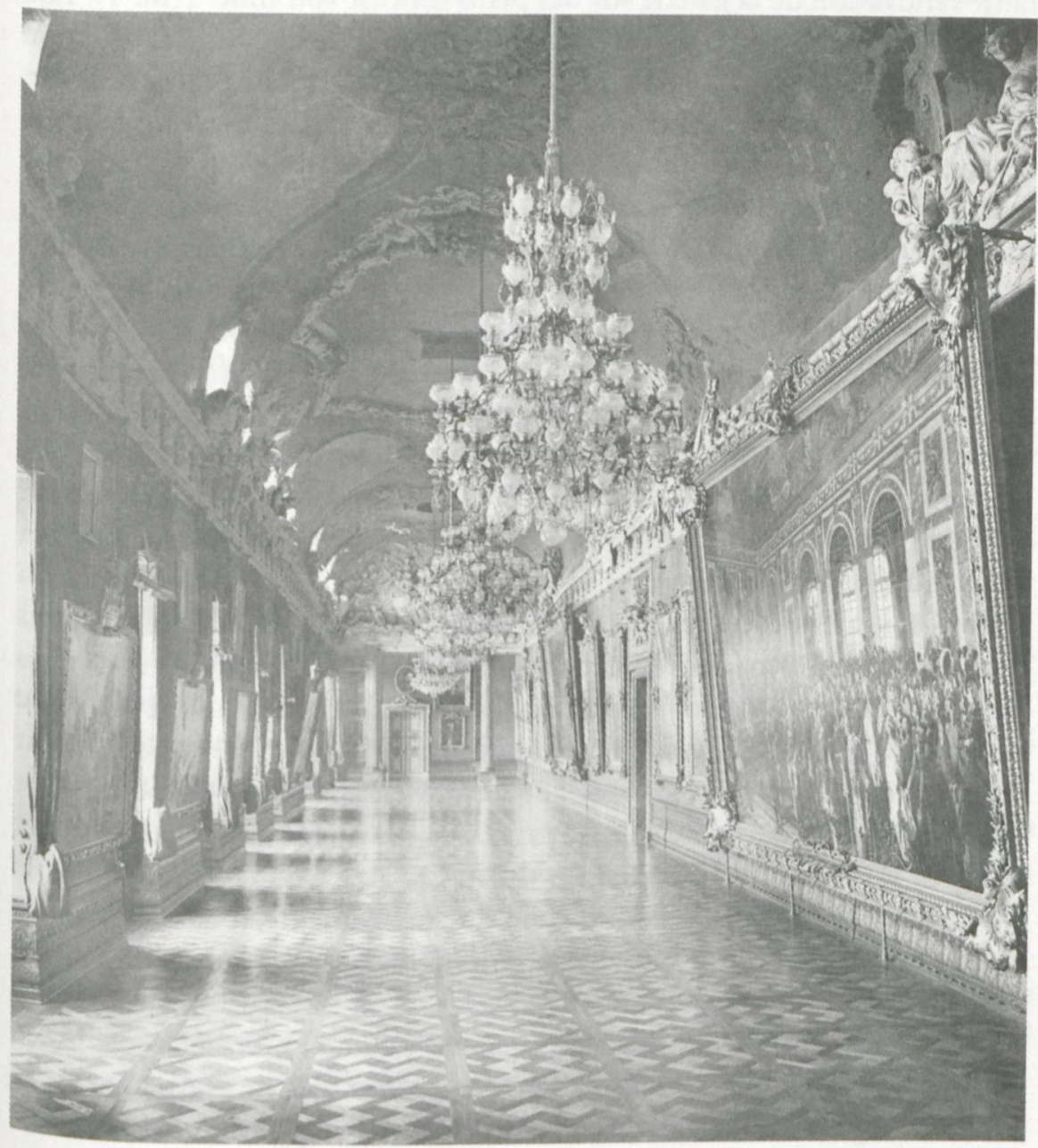

III. 5, Vue de la Grande Galerie (alors Gemäldegalerie) vers l'est, avant 1901, au premier plan à droite, on peut voir la peinture d'Anton von Werner,

La proclamation de l'empereur allemand dans la galerie des Glaces de Versailles, 1877.

61. Hennings, c. $1905: 28-33$. 
L'aménagement sous Guillaume II : de la galerie de Peintures à la galerie dite des Gobelins

De 1913 à 1916, Guillaume II commanda une nouvelle et importante rénovation de la galerie sur des plans d'Ernst von Ihne ${ }^{62}$ (Ill. 1). Elle fut dès lors requise en priorité pour des festivités de la cour, avec la Salle blanche, qui avait été intégralement réaménagée quelques années plus tôt. L'étoffe rouge tendue sur les murs fut retirée et remplacée par un lambris de chêne ouvré de ciselures néo-baroques dans un style qui reproduisait aussi fidèlement celui de l'époque de la création de la galerie que la peinture centrale, Glorification des armoiries prussiennes, ajoutée au plafond par Martin Frost, copie d'une œuvre réalisée en 1700 par Augustin Terwesten pour la deuxième antichambre d'apparat. Les peintures rouges sur fond rouge ajoutées vers 1835 en face des fenêtres des mezzanines furent remplacées par des miroirs muraux. Parce que la collection de peintures du XIX ${ }^{e}$ siècle contrariait l'effet d'ensemble baroque, presque tous les tableaux furent retirés et remplacés par six tapisseries de grand format de Pierre Mercier réalisées à l'époque de Frédéric ${ }^{\mathrm{er}}$, illustrant des batailles du grand électeur. Ces tapisseries, qui provenaient à l'origine du château berlinois, avaient été ensuite exposées au musée des Hohenzollern dans le château de Monbijou, où furent transférées en contrepartie les œuvres majeures de la collection ${ }^{63}$. La diversité formelle et thématique fut donc sacrifiée au profit d'un arrangement homogène et ordonné qui estompait la différence entre le baroque original et les éléments néo-baroques. Cet agencement fut celui de la galerie jusqu'à la destruction du château entre 1945 et 1950 . Après l'installation du musée des Arts décoratifs dans le château en 1920, la salle connut peu de changements, à l'exception des nombreuses vitrines dressées du côté des fenêtres dans lesquelles étaient exposés des verres historiques ${ }^{64}$.

Au cours de deux siècles, cette galerie s'était donc métamorphosée d'une galerie des Glaces jamais achevée en une galerie d'Argent, de la galerie d'Argent en une galerie de Peintures, et de la galerie de Peintures en

62. Geyer, 1992, vol. texte: 118-119.

63. Kemper, $2005: 114-116$.

64. Führer durch das Schloßmuseum, c. 1926: 16-17. 
une galerie dite des Gobelins ${ }^{65}$. Si la reconstruction du château de Berlin sous son apparence originale est entreprise à partir de 2010, celle de la galerie n'a pas encore été envisagée, bien que les six tapisseries de la manufacture de Pierre Mercier aient été conservées. Parce qu'elle relève d'un concept artistique singulier, c'est cette dernière phase, la galerie des Gobelins de Guillaume II, qui mériterait d'être retenue comme base d'une éventuelle reconstruction ${ }^{66}$.

\section{Bibliographie}

Arminjon, Catherine (dir.), 2007, Quand Versailles était meublé d'argent, cat. expo. (château de Versailles, 19 novembre 2007 au 9 mars 2008), Versailles-Paris, Établissement public du musée et du domaine national de Versailles-Éditions de la Réunion des musées nationaux.

BAEDEKer, Karl, 1878, Berlin, Potsdam und Umgebungen: separat-Abdruck aus Baedeker's Nord-Deutschland, Leipzig, Baedeker.

- 1887, Berlin und Umgebungen: Handbuch für Reisende, Leipzig, Baedeker.

BAER, Winfried, 2007, "Au Château Royal de Berlin ", in C. Arminjon (dir.), Quand Versailles était meublé d'argent, cat. expo. (château de Versailles, 19 novembre 2007 au 9 mars 2008), Versailles-Paris, Établissement public du musée et du domaine national de VersaillesÉditions de la Réunion des musées nationaux : 125-141.

BarTMann, Dominik (dir), 1993, Anton von Werner: Geschichte in Bildern, cat. expo. (Berlin, Deutsches Historisches Museum, 7 mai au 27 juillet 1993), Munich, Hirmer Verlag.

B ARTOSCHEK, Gerd; VOGTHERR, Christoph Martin (dir.), 2004, Zerstört, entführt, verschollen: Die Verluste der preußischen Schlösser im Zweiten Weltkrieg: Gemälde I, Potsdam, Stiftung Preußische Schlösser und Gärten Berlin-Brandenburg.

65. Ce nom de galerie des Gobelins qui s'est alors imposé est erroné au sens strict, puisqu'il

$\mathrm{s}^{\prime}$ agit de tentures murales de fabrication berlinoise.

66. Hinterkeuser, $2006: 38-39$. 
Berliner geschriebene Zeitungen aus den Jahren 1713 bis 1717 und 1735: ein Beitrag zur Preußischen Geschichte unter König Friedrich Wilhelm I., 1902, édité par E. Friedlaender, Berlin, Verein für die Geschichte Berlins.

BoECK, Wilhelm, 1933, "Les sculpteurs français à la cour de Frédéric I ${ }^{\text {er }}$ de Prusse ", Gazette des Beaux-Arts, 75/2 : 104-116.

Borrmann, Richard, 1893, Die Bau- und Kunstdenkmäler von Berlin, Berlin, J. Springer.

Die Bildergalerie in Sanssouci: Bauwerk, Sammlung, und Restaurierung: Festschrift zur Wiedereröffnung 1996, 1996, Berlin-Milan, Generaldirektion der Stiftung Preussische Schlösser und Gärten Berlin-Brandenburg-Skira.

Führer durch das Schloßmuseum: im Auftrage des Generaldirektors der Staatlichen Museen, c. 1926, Berlin, Reichsdruckerei.

Gaehtgens, Thomas W., 1990, Anton von Werner: die Proklamierung des Deutschen Kaiserreiches: ein Historienbild im Wandel preußischer Politik, Francfort-sur-le-Main, Fischer Taschenbuch Verlag.

GEYER, Albert, 1936, Geschichte des Schlosses zu Berlin, t. I : Die kurfürstliche Zeit bis zum Jahre 1698, Berlin, Deutscher Kunstverlag, 2 vols.

- 1992, Geschichte des Schlosses zu Berlin, t. II : Vom Königsschloß zum Schloß des Kaisers (1698-1918), Berlin, Nicolai Verlag, 2 vols.

Hennings, E., c. 1905, Das königliche Schloss in Berlin: ein Führer durch seine Sehenswürdigkeiten, Berlin, à titre d'auteur.

HinterkeUSER, Guido, 2001, "Blick nach Europa: die Architektur in Berlin im Zeitalter Friedrichs III./I. ", in Preußen 1701: eine europäische Geschichte, cat. expo. (Berlin, Schloss Charlottenburg, 6 mai au 5 août 2001), Berlin, Stiftung Preussische Schlösser und Gärten, vol. 2 : 254-268.

- 2003, Das Berliner Schloss: der Umbau durch Andreas Schlüter, Berlin, Siedler.

- 2005-2006, «Die Wohn- und Prunkräume Sophie Charlottes und Friedrich I. im Schloß Charlottenburg: zu Programmatik, Ausstattung und Nutzung ", Zeitschrift des Deutschen Vereins für Kunstwissenschaft, 59-60: 243-268.

- 2006, Das Berliner Schloss-mehr als nur Fassade: die verlorenen Innenräume des Berliner Schlosses und die Möglichkeiten der Rekonstruktion zerstörter Raumkunst nach 1945, Munich, Deutscher Kunstverlag. 
- (à paraître), "Les pièces d'habitation et les salles d'apparat de Sophie Charlotte et Frédéric I ${ }^{\text {er }}$ au château de Charlottenburg: finalité, aménagement et utilisation ", in T. W. Gaehtgens, M. A. Castor et F. Bussmann (dir.), Versailles et l'Europe : l'appartement royal et princier, architecture, décor, cérémonial, Paris, Éditions de la Maison des sciences de l'homme.

Holland, Alexander, 2002, Johann Friedrich Eosander genannt von Göthe (1669-1728): Anmerkungen zu Karriere und Werk des Architekten, Ingenieurs und Hofmannes am Hof Friedrichs I. in Preussen, Weimar, Verlag für Datenbank und Geisteswissenschaften.

KEISER, Johann, 1749, Kurtze Beschreibung der königl. und churf. Hauptund Residentz Berlin, t. II : Von d. alten Schloße Müntzthurm u. neue Schloße, Berlin, s. n.

KEMPER, Thomas, 2005, Schloss Monbijou: von der königlichen Residenz zum Hohenzollern-Museum, Berlin, Nicolai Verlag.

Klưnner, Hans-Werner, PeschKen, Goerd, 1982, Das Berliner Schloss: das klassische Berlin, Francfort-sur-le-Main, Propyläen.

Kommer, Björn R., 1974, Nicodemus Tessin der Jüngere und das Stockholmer Schloss: Untersuchungen zum Hauptwerk des schwedischen Architekten, Heidelberger, C. Winter.

LADENDORF, Heinz, 1935, Der Bildhauer und Baumeister Andreas Schlüter: Beiträge zu seiner Biographie und zur Berliner Kunstgeschichte seiner Zeit, Berlin, Deutscher Verein für Kunstwissenschaft.

MABILLE, Gérard, 2007, "Le mobilier d'argent de Louis XIV ", in C. Arminjon (dir.), Quand Versailles était meublé d'argent, cat. expo. (château de Versailles, 19 novembre 2007 au 9 mars 2008), VersaillesParis, Établissement public du musée et du domaine national de Versailles-Éditions de la Réunion des musées nationaux : 61-83.

Morin, Friedrich, 1860, Berlin und Potsdam im Jahre 1860: Neuester Führer durch Berlin, Potsdam und Umgebungen. Ein Taschenbuch für Einheimische und Fremde, Berlin, Nicolaische Verlagsbuchhandlung.

Mưller, Johann Christoph; Küster, Georg Gottfried, 1756, Altes und Neues Berlin, partie III, Berlin, J. P. Schmid.

NICOLAI, Friedrich, 1786, Beschreibung der Königlichen Residenzstädte Berlin und Potsdam: aller daselbst befindlicher Merkwürdigkeiten, und der umliegenden Gegend, Berlin, F. Nicolai. 
Peschken, Goerd, 1992, Das königliche Schloss zu Berlin, t. I : Die Baugeschichte von 1688-1701, Munich, Deutscher Kunstverlag.

Peschken, Goerd, Wiesinger, Liselotte, 2001, Das königliche Schloss zu Berlin, t. III : Die barocken Innenräume, Berlin-Munich, Deutscher Kunstverlag, 2 vols.

REINHARD von, Marianne, 1852, Berlin: ein Buch für junge und alte Preußen, Berlin, A. Duncker.

RuMPF, Johann Daniel Friedrich, 1794, Beschreibung Der Aeussern Und Innern Merkwürdigkeiten Der Königlichen Schlösser in Berlin, Charlottenburg, Schönhausen, in Und Bey Potsdam, Berlin, Oehmigke.

SCHASLER, Max, 1856, Berlins Kunstschätze: ein praktisches Handbuch zum Gebrauch bei der Besichtigung derselben, vol. 2 : Die öffentlichen und Privat-Kunstsammlungen, Kunstinstitute und Ateliers der Künstler und Kunstindustriellen von Berlin [...] Künstlerateliers u.s.f., Berlin, F. Nicolai.

Schramm, Carl Christian, 1744, Neues Europäisches Historisches ReiseLexikon, Leipzig, J. F. Gleditsch.

SeIDEL, Paul, 1896, Der Silber- und Goldschatz der Hohenzollern im Königlichen Schlosse zu Berlin, Berlin, Cosmos.

StrunCK, Christina, 2007, Berninis unbekanntes Meisterwerk: die Galleria Colonna in Rom und die Kunstpatronage des römischen Uradels, Munich, Hirmer.

Verzeichniß von Gemälde und Kunstwerken, welche durch die Tapferkeit der vaterländischen Truppen wieder erobert worden und [...] öffentlich ausgestellt sind, 1815, Berlin, Quien.

VogTHERr, Christoph Martin, 2005, "Die Auswahl von Gemälden aus den preußischen Königsschlössern für die Berliner Gemäldegalerie im Jahr 1829 ", Jahrbuch der Berliner Museen, vol. 47 : 63-105.

WIESINGER, Liselotte, 1992, Deckengemälde im Berliner Schloss, Francfortsur-le-Main, Propyläen.

Wilhelmine, Friederike Sophie, 1967, Mémoires de Frédérique Sophie Wilhelmine, Margrave de Bayreuth, sour de Frédérique le Grand, depuis l'année 1706 jusqu'à 1742, écrits de sa main, notes de G. Doscot, Paris, Mercure de France. 